\title{
EL ENCUENTRO ENTRE LÓGICAS GLOBALES Y LOCALES: EMPLEO BANANERO Y TURÍSTICO EN CAHUITA Y PUERTO VIEJO*
}

\author{
THE MEETING AMONG LOCAL AND GLOBAL LOGICS: BANANA \\ EMPLOYMENT AND TOURISM IN CAHUITA AND PUERTO VIEJO
}

\author{
Eugenia Molina Alfaro**
}

RESUMEN

En este artículo se propone que existen lógicas sociales que son históricas y que determinan los alcances del desarrollo sobre determinados territorios o localidades. Para entender el desarrollo actual en el caso de Cahuita y Puerto Viejo en el Caribe sur de Costa Rica, entre las lógicas socio-históricas más importantes se destaca el papel que han jugado las actividades productivas, el desarrollo de las comunicaciones, la generación de empleo, la migración y la expansión turística. A partir de una conceptualización de estas lógicas como formas de estudiar las nuevas relaciones entre lo local y lo global, se describen y analizan algunos de los impactos que ha tenido la expansión turística en las comunidades de Cahuita y Puerto Viejo del Caribe Sur costarricense.

PALABRAS CLAVES: LIMÓN, CR * DESARROLLO LOCAL * GLOBALIZACIÓN * PRODUCCIÓN BANANERA $*$ TURISMO $*$ EMPLEO

\section{ABSTRACT}

This article proposes that there are different types of social logics that determine the development over specific territories or communities. This logic is historical and to understand today the development in Cahuita and Puerto Viejo on the southern Caribbean coast of Costa Rica, among the most important socio-historical logics one must consider the role of production activities, development of communications, employment, migration patterns and tourism expansion. Beginning with the conceptualization of these logics as ways to study the new relationships between the local and the global, the impacts that tourism expansion has had in Cahuita and Puerto Viejo are described and analyzed.

KEY WORDS: LIMÓN, CR * LOCAL DEVELOPMENT * GLOBALIZATION * BANANERA PRODUCTION $*$ TOURISM $*$ EMPLOYMENT

Este artículo es un producto del proyecto de investigación titulado "El potencial de los espacios locales para la resolución de problemas del desarrollo y la conformación de identidades" del Instituto de Investigaciones Sociales de la Universidad de Costa Rica.
** Investigadora. Instituto de Investigaciones Sociales, Universidad de Costa Rica. molina.eugenia@gmail.com 


\section{PUNTO DE PARTIDA CONCEPTUAL Y METODOLÓGICO}

Para algunos autores (Wallerstein, 1979) el contexto internacional actual remite a una creciente economía global donde los Estados nacionales, ante el ímpetu de las empresas transnacionales, han perdido el monopolio de su capacidad regulativa en lo político y en lo económico. Para otros (Portes y Kincaid, 1990), el Estado continúa manteniendo la centralidad en los procesos nacionales. Pero en cualquiera de los casos hay coincidencia de que se ha producido una revitalización de lo local en el contexto presente. Este documento se plantea qué significa y cómo estudiar esos procesos de revitalización de lo local.

Hay dos aproximaciones frecuentes en el abordaje de las comunidades como objeto de estudio, que son las visiones historicistas y los enfoques estructuralistas sobre lo local. En el primer caso la definición de lo local estuvo por mucho tiempo asociada a contenidos próximos a la autarquía. En esa perspectiva, la comunidad se visualizaba como expresión de un lugar único del cual interesaba rescatar su especificidad y muchos estudios agotaron su análisis en la descripción de la comunidad en sí misma. Se rescató la historia local pero haciendo caso omiso de las relaciones de dicha localidad con su entorno nacional inmediato y con el contexto internacional.

La segunda aproximación a lo local la aporta el enfoque estructuralista $y$ en esta perspectiva la comunidad puede verse como una sociedad micro donde se espera encontrar $y$ estudiar la forma en que se reproducen las contradicciones y procesos más generales del todo social. En esta perspectiva, los estudios de comunidades sirven para ejemplificar tendencias más generales y a diferencia de los anteriores, los autores tienden a perder de vista la visión histórica cayendo en aproximaciones únicamente sincrónicas con instrumentos tales como encuestas.

Esta investigación toma como punto de partida un enfoque intermedio entre los dos anteriores y es aquél que ve lo local como forma idónea para el abordaje de las relaciones entre lo interno $y$ lo externo, lo particular y lo universal, lo estructural y lo coyuntural, así como también entre lo viejo y lo nuevo. De esta forma se busca no sobredimensionar lo singular ni tampoco aproximarse a la comunidad como un lugar de reproducción de lo universal, sino que más bien el centro del análisis es precisamente la relación entre lo específico de la comunidad y los procesos más generales de la sociedad actual.

Para esto se han retomado un conjunto de aportes conceptuales que se encuentran en el planteamiento sobre desarrollo local de Arocena (1995), en el concepto de glocalización propuesto por Robertson (1995) y en la propuesta analítica de Pérez Sainz (2000) sobre comunidades centroamericanas globalizadas.

En forma sucinta se puede decir que, en el estudio del desarrollo local, tanto Arocena como Pérez Sainz consideran como centrales las lógicas históricas aludiendo por ellas a los componentes específicos que distinguen un territorio $y$ en particular a los elementos de identidad sociocultural que lo definen. Ambos autores coinciden también en que es necesario analizar cada localidad ubicándola dentro de un sistema mayor del cual forman parte. Mientras Arocena se refiere a esto como lógicas sistémicas, Pérez Sainz plantea que tales lógicas en el contexto actual son las lógicas de la globalización.

Un tercer tipo de lógicas definitorias de lo local serían las que remiten al poder, el estado y las relaciones entre actores locales. Mientras Arocena alude a ese tipo de lógica con el concepto de sistema de actores; Pérez Sainz habla de la importancia de las lógicas estatales en la definición de lo local pero, también destaca nuevos actores relevantes en el proceso de globalización. En todo caso, uno de los elementos centrales que distinguen la teoría del desarrollo local respecto de otras teorías del desarrollo es que releva el papel de los actores en el cambio social, tratando de superar las visiones más estructuralistas que dejan poco espacio a la acción de los agentes sociales frente al comportamiento de las estructuras socioeconómicas.

Por su parte, Robertson ha planteado que lo global y lo local no se excluyen mutuamente por cuanto lo glocal es una forma de reconocer 
que lo segundo debe entenderse como un aspecto de lo primero. Beck retoma esa perspectiva $y$ agrega que "[...] la globalización [...] es asible en lo pequeño y lo concreto, in situ, en la propia vida $y$ en los símbolos culturales, todo lo cual lleva al sello de lo glocal" (Beck, 1997:80).

Estos tres autores permiten trazar algunos elementos claves para el estudio de la relación entre lo global y local de manera que puedan identificarse lógicas claves que impactan un territorio específico, tales como:

a) Lógicas históricas, que pueden estar referidas a los elementos identitarios de la comunidad y a la relación entre factores internos $y$ externos que inciden sobre el territorio.

b) Ubicar lo pequeño dentro lo grande, es decir analizar las lógicas sistémicas dentro de las cuales se sitúa la comunidad. Esto puede hacerse estableciendo relaciones entre la comunidad $y$ un territorio más amplio como la nación o la economía mundial. Pero también supone establecer relaciones de determinación entre diversos ámbitos tales como la economía, la cultura o la naturaleza.

c) Lógicas políticas, referidas al papel que juegan el estado y los diversos actores que inciden dentro de un territorio.

Con este punto de partida conceptual se realizó una investigación que trata de identificar algunas de las principales lógicas que han impactado las comunidades de Puerto Viejo y Cahuita en el Caribe Sur. Para ello se ha tratado de combinar una reconstrucción histórica del distrito, con una ubicación en un territorio y sistema más amplio $y$, también con un análisis sincrónico del empleo generado por la actividad turística en el periodo reciente. De esta forma se busca trascender el estudio de caso descriptivo y se presentan en este documento algunas de las lógicas más impactantes sobre el territorio.

En el marco de esta investigación se realizaron dos encuestas y diez entrevistas a profundidad. La primera se aplicó a 89 asalariados de negocios turísticos de Puerto Viejo y Cahuita en el periodo transcurrido entre julio $y$ agosto del año 2001; la segunda, se aplicó a 151 hogares de Puerto Viejo en octubre del año $2003^{1}$.

\section{DE LA ACTIVIDAD AGRÍCOLA A LOS SERVICIOS TURÍSTICOS}

En este apartado se exponen dos argumentos centrales: primero que, hasta fines de la década del setenta del siglo pasado, Cahuita y Puerto Viejo constituyeron sociedades locales eminentemente rurales cuya base económica y social estuvo ligada a la producción agrícola. Y segundo, se arguye que a partir de los años ochenta $y$ noventa, el distrito de Cahuita, sufre un proceso de diferenciación social y territorial asociada directamente con la expansión de la actividad turística que se constituyó en el polo de desarrollo económico más dinámico.

Las comunidades de Cahuita y Puerto Viejo constituyen los dos centros más poblados del distrito de Cahuita, que tiene una extensión de 170,4 km² y una población de 5331 habitantes. Este distrito forma parte del cantón de Talamanca, creado en mayo de 1969, cuya extensión representa el 5\% del territorio nacional $y$ constituye el menos poblado (25 857 habitantes) y de menor densidad $\left(9 \mathrm{~h} / \mathrm{km}^{2}\right)$ de los cantones de la Región Huétar Atlántica.

Los cambios más importantes de Cahuita y Puerto Viejo están relacionados con la forma en que fenómenos internacionales como la migración, la expansión de la industria bananera y luego el turismo se han expresado sobre la geografía de la región. De igual forma, se puede decir que han sido las características geográficas factores esenciales en la atracción y expulsión de las poblaciones y las inversiones en la región. En este cantón pueden distinguirse tres zonas:

a) la Alta Talamanca que constituye la parte montañosa donde se refugiaron los indígenas

1 Una de las encuestas mencionadas fue diseñada y aplicada por la autora, junto con Michael Herradora y Edgar Nuñez en el marco del Diplomado Superior de Investigación del Desarrollo Económico Local de la FLACSO Costa Rica; dirigido por Juan Pablo Pérez Sáinz. 
durante la colonización española y luego tras la llegada posterior de las transnacionales bananeras,

b) una parte intermedia de colonización tardía que se encuentra entre la zona montañosa y la costa, donde se asentaron inmigrantes de distintas partes del país en la década del sesenta y setenta (Alvarado, 1994:21) y

c) la tercera zona constituye la faja costera del cantón. Esta última fue la primera en colonizarse $y$ es donde se encuentran ubicadas las dos comunidades de estudio.

Mientras en la primera zona predomina la población indígena en las dos últimas, hasta fines de los años setenta, predominaba la población de origen antillano y algunos latinos incluidos los inmigrantes blancos costarricenses.

A grandes rasgos se puede señalar que las comunidades de estudio han formado parte de diversas configuraciones territoriales tales como:

$\diamond$ campamentos provisionales de la casi despoblada comarca limonense y luego pequeños caseríos y poblados de indígenas $y$ afrocaribeños que se ubican en las costas a fines del siglo XIX,

$\diamond$ el enclave bananero establecido por las transnacionales norteamericanas durante $1880-1950^{2}$,

४ las pequeñas comunidades y colonias agrícolas de los productores de cacao 19601970 ,

$\diamond$ una micro-región turística que se configura lentamente en el periodo 1980-2000.

Se sabe que el mismo gobierno costarricense promovió la colonización de la zona desde fines del siglo XIX (Viales, 2001:74). Y como narra Palmer, desde ese entonces hubo

Se tomó el año 1883 por ser el de la primera exportación de banano pero hay diversas periodizaciones según los diferentes autores. Viales, 1998 por ejemplo, retoma el año 1927 como el inicio del descenso de la producción bananera y el periodo 1927-1950 para estudiar la crisis que provocó el enclave cuando la compañía bananera se trasladó del Atlántico al sur del país. inmigrantes de Bocas del Toro, la costa miskita nicaragüense y de San Andrés de Colombia, que venían a pescar y se instalaban en la costa por temporadas (Palmer, 2000: 42). Más tarde esas $y$ otras poblaciones inmigrantes dieron origen a los primeros poblados $y$ a la agricultura de subsistencia con la piña, la sandía, la yuca, la caña y el coco entre sus principales provisiones (Palmer, 2000: 25).

Desde el punto de vista de la configuración socioterritorial que prevaleció en la zona, diversos autores coinciden en que durante el periodo 1880-1950 el enclave bananero irradió toda la región atlántica (Viales, 1998: 25-30). El concepto de enclave remite al dominio concedido por el estado, al capital de origen foráneo sobre una unidad territorial productiva que desarrolla escasos vínculos con la economía nacional y local. En ese sentido, se puede decir que en ese lapso de tiempo, socio territorialmente hablando Cahuita y Puerto Viejo formaron parte de una gran plantación bananera ${ }^{3}$.

La expansión bananera en nuestro país estuvo asociada con la construcción del ferrocarril al Atlántico pues como parte de las concesiones para la construcción de este, el gobierno otorgó grandes extensiones de tierra para el cultivo de banano al consorcio de lo que llegaría a ser la United Fruit Company (UFCO) creada en 1889. Trabajadores de Jamaica, Barbados y Saint Kitts, fueron traídos desde sus países para la construcción de la línea ferroviaria y para trabajar en la misma plantación bananera. Ya en 1939 el cantón de Talamanca contaba con aproximadamente 3000 pobladores (Viales, 1998:146).

La UFCo generó diversos vínculos productivos con la economía local. Por un lado, algunos lugareños pasaron de ser productores a empleados de la compañía o le arrendaron terrenos a esta para cultivar banano y cacao. Por otro, se amplió el mercado para los productos tradicionales. Como en ese entonces únicamente era ella la que compraba la producción de las

3 Existe una discusión en torno al concepto de enclave. Mientras Casey, 1979 señala que todas las plantaciones bananeras siguieron un patrón, Viales, 1998 plantea las especificidades de la zona atlántica costarricense. 
pequeñas fincas, se producía un dominio total en la fijación de precios mientras que los productores locales eran los que asumían la totalidad del riesgo de la producción ${ }^{4}$. Precisamente por esto, desde fines de los años treinta el inicio de la salida de dicha compañía ocasionó un período crítico en la economía local. Los pobladores tuvieron que volver a depender solo del cacao, producto que la misma compañía había introducido desde 1913 y se convirtió en la principal fuente generadora de ingresos especialmente para la población negra (Viales, $1998: 130)^{5}$. No obstante, ya en 1967, la compañía no producía ni banano ni cacao (Bourgois, 1994:34). Con la partida de la empresa quedaron disponibles grandes cantidades de tierra $y$ de personas desempleadas, $y$ por primera vez se asentaron en la zona cantidades importantes de personas de habla hispana (Palmer, 2000: 169). Con la crisis bananera Talamanca fue uno de los cantones que quedan aislados, marginados e incomunicados del resto de la región. Hay registros que evidencian cómo en 1938 sus pobladores rogaban (literalmente) al gobierno para que desarrollara una colonia agrícola, una vía telefónica y una vía de transporte entre Cahuita y Limón (Viales,1998: 85,145). En ese contexto el gobierno promueve la neocolonización y experimenta con distintos proyectos, tanto productivos como de explotación petrolera y maderera ${ }^{6}$.

Dentro del mismo distrito hubo un período de ocupación por parte de compañías transnacionales ya que en 1930 la Penhurst Banana Company obtuvo una concesión para explotar las tierras ubicadas en lo que hoy es conocido como Hone Creek.

A fines de los años setenta su precio llegó a 1500 colones el quintal alrededor de $\$ 175$ de la época y según datos del Censo Agropecuario de 1973, el $83 \%$ de la producción nacional de este cultivo era generado en la Región Huetar Atlántica. De esta cantidad, el $74 \%$ era cultivado en el cantón de Talamanca. Así, el cacao llegó a representar el 90\% del ingreso económico de la región (Palmer, 2000: 180).

Así, a inicios de la década del 50 el Estado costarricense facilitó una concesión para realizar explotaciones petroleras en Talamanca a la Compañía Loffland, la cual estuvo en la región aproximadamente tres años. Las instalaciones de la empresa estuvieron ubicadas
La producción de subsistencia toma fuerza también en este periodo pues anteriormente la misma Compañía suplía los requerimientos básicos. Como señala Viales, 1998 en "la parte negra de Limón" los cultivos de yuca, plátanos y ñame jugaron un papel importante como parte de la dieta básica de la población de origen antillano ${ }^{7}$.

Después del año 1956 se produce el retorno de las multinacionales a la región atlántica, quienes continúan con el desplazamiento ya iniciado tiempo atrás, de la producción en los productores nacionales $y$ asumiendo ellas el control sobre la comercialización (Carcanholo, 1978:194). En el caso de la división bananera de Talamanca, es decir la ubicada entre Bocas del Toro en Panamá y el distrito de Sixaola en Costa Rica, la producción bananera se reactivó hasta después del año 1978 (Bourgois, 1994:34).

En la década del setenta se produjeron tres hechos que tuvieron fuertes repercusiones sobre la configuración territorial de las dos comunidades de estudio: la creación del Parque Nacional Cahuita, en 1970; la apertura de la

en Puerto Viejo. La presencia de esta empresa facilitó la construcción del camino hasta la comunidad de Patiño en donde se encontraba un sitio de exploración, y posteriormente avanzó hasta Bribrí (antiguo Fields) y hasta Shiroles; $y$ también contribuyó al mejoramiento del campo de aterrizaje que existía en Puerto Viejo. Posteriormente, estuvo también la Gulf Oil Company, y dos décadas después hubo otros intentos de exploración petrolera por parte de la Refinadora Costarricense de Petróleo y por la empresa mexicana Petróleos de México (PEMEX); sin embargo, ninguna de ellas encontró cantidades comercializables del preciado líquido.

$7 \quad$ Durante el periodo $1932-1950$ se identifican importantes esfuerzos del gobierno y los actores regionales limonenses por encontrar alternativas productivas para la zona pero la mayoría de estas fracasan. En el caso de Talamanca el cacao resultó ser el cultivo más permanente y rentable para la zona. Sin embargo, a finales de los setenta, la enfermedad de la monilia lo atacó provocando el abandono de muchas fincas dedicadas a este cultivo y con ello una crisis que hizo más clara la necesidad de la diversificación productiva (Fonseca, 1999: 213). 
vía terrestre que comunica las comunidades de estudio con Puerto Limón y con San José, en 1976 y la reactivación de la producción bananera. Las opciones laborales para los pobladores volvieron a ser las siguientes: a) vivir de la producción de subsistencia en su tierra, b) producir banano y venderlo a las compañías nacionales o extranjeras o c) emplearse como asalariado parcial o totalmente para las compañías bananeras.

La segunda tesis planteada al inicio de este apartado es que a pesar del peso que aún tiene el sector agrícola dentro del distrito de Cahuita, $y$ debido al dinamismo que presenta la expansión turística en las últimas dos décadas, esta se ha transformado en una sociedad local de base turística. Para entender cómo se produjo esa transformación conviene mencionar algunos elementos claves.

El decreto ejecutivo de setiembre de 1970, mediante el cual el gobierno hacía de gran parte del distrito de Cahuita, un área protegida provocó gran conflictividad en la misma comunidad. Esto porque para los pobladores la iniciativa fue vista como una imposición del gobierno e implicaba el desalojo arbitrario de muchos de ellos y la prohibición de sus actividades tradicionales de agricultura de cacao, coco, cacería y pesca. Después de más de una década de conflictos entre el Estado $y$ los pobladores se llega a algunos acuerdos básicos que le permiten a los primeros mantener sus actividades económicas para la sobrevivencia. No obstante lo anterior, la producción de cacao, su cultivo principal hasta entonces, también entró en crisis al ser atacada por el hongo de la monilia a fines de los setenta e inicios de los ochenta. Con el debilitamiento de este como alternativa productiva y con la pérdida de las tierras del parque, el turismo se fue quedando como una nueva y casi única alternativa rentable (Fonseca, 1999: 221).

Aunque el conflicto aún no termina, puesto que no todos los terrenos expropiados han sido cancelados por el Estado, se puede decir que mediante la creación del Parque Nacional se promovió la llegada de nuevos visitantes e inmigrantes que, poco a poco transformaron las dos comunidades de estudio en una micro-región turística que va desde Cahuita, pasando por Puerto Viejo hasta GandocaManzanillo.

Entre los cambios más visibles está el desplazamiento de la población afrocaribeña con la llegada de europeos y norteamericanos a residir en la zona. Este factor ha transformado el espacio en un conglomerado de pequeños y medianos negocios turísticos (cluster) ${ }^{8}$ que sólo entre Cahuita y Puerto Viejo asciende a 150, entre restaurantes, hospedajes, oficinas de información turística, ventas de artesanía, entre otros contabilizados en agosto del año 2001. Mientras en Puerto Viejo el porcentaje de dueños de negocios turísticos extranjeros es del 36\% en Cahuita asciende al 65\% (Molina y otros, Encuesta de establecimientos: 2001).

Un rasgo relevante de la nueva configuración espacial es el doble carácter que asume la expansión de la actividad turística; por un lado, se revitalizan ciertas comunidades costeras, mientras que por el otro se deprimen $y$ quedan marginados otros poblados que no gozan de los atractivos turísticos, tales como Punta Riel o Comadre, Hone Creek, San Rafael y Carbón.

Esto también contribuyó al desarrollo de una nueva actividad económica de la cual dependen Cahuita y Puerto Viejo y a través de la cual, estas se han posicionado a escala nacional e internacional como destinos turísticos. Llegando a ser en la década del noventa el motor de la actividad económica del distrito de Cahuita. Las poblaciones de los centros de Puerto Viejo y Cahuita han logrado producir una diferenciación del servicio en contraste con el que ofrece la región de Guanacaste captando también un tipo de turista diferente. No sólo han construido una oferta original a partir de la cultura indígena y afrocaribeña (etnia, lenguaje, cocina, música) sino también a través del manejo que se hace de los recursos ambientales de su bosque tropical húmedo, en contraste también con el bosque seco guanacasteco. De esta forma Puerto Viejo aprovecha sus recursos naturales pues cuenta con ocho kilómetros de costa frente al mar Caribe y está rodeado de reservas naturales. Las principales son el Parque Nacional Cahuita, el Refugio Nacional de Vida Silvestre Gandoca-Manzanillo y la Reserva Biológica Hitoy-Cerere. 


\section{EL EMPLEO EN CAHUITA: DE LA FALTA DE OPORTUNIDADES A LA PRECARIEDAD LABORAL}

Más allá de las percepciones de los pobladores sobre el turismo ${ }^{9}$ se toma como punto de partida en este artículo la relevancia atribuida a las lógicas del empleo para entender los procesos de integración y exclusión comunitaria en el contexto centroamericano actual (Sojo y Pérez, 2000:15) y desde esa visión, se presentan dos argumentos. El primero de ellos es que la expansión del turismo ha conllevado una mejora relativa importante, desde una perspectiva histórica, en la calidad del empleo del cantón de Talamanca y concretamente del distrito de Cahuita. Y en segundo lugar, no obstante lo anterior, el empleo generado por la actividad turística puede considerarse un empleo precario. Aunque escapa de las posibilidades de este documento exponer una comparación exhaustiva entre el empleo bananero y el turístico una caracterización general de las condiciones laborales que generan ambos tipos de actividad pueden aportar luces al respecto.

\subsection{DEL DESGASTE FÍSICO, LA EXPLOTACIÓN Y LA FALTA DE OPORTUNIDADES}

De forma muy sintética, se puede decir que antes de la década del cuarenta las condiciones de contratación en las bananeras eran infrahumanas ${ }^{10}$. Ni siquiera con la gran huelga bananera del Atlántico, en 1934 lograron los

En una encuesta que realizó la autora en 151 hogares de Puerto Viejo, en el año 2003, se les preguntó a los pobladores acerca de cuáles eran los cambios principales que habría traído la expansión turística $y$ los principales que se señalaron fueron los relacionados con el territorio y con la situación económica. Además, el $60 \%$ de los encuestados valoraron positivamente el impacto del turismo en la comunidad. Surge la pregunta de ¿si ambas respuestas recogen cierta visión histórica sobre lo que ha sido el empleo generado en la costa?

Con respecto al periodo inicial del gran auge de la exportación bananera 1880-1914, muchos autores han evidenciado las condiciones infrahumanas a las que se sometieron los trabajadores (Bourgois, 1994). trabajadores abolir el trabajo a destajo, elevar los bajos salarios y mejorar las condiciones de vida. Esas luchas verían sus frutos una década más tarde cuando en 1944 se aprueba la legislación laboral que establece el derecho de organización y una serie de regulaciones para el trabajo y la seguridad social de los trabajadores. Es a partir de los años cincuenta que la jornada de ocho horas, el salario mínimo y el acceso al seguro social fueron reguladas por el estado y se aplican a la expansión bananera que, se ha mantenido con distintos altibajos pero con bastante continuidad hasta hoy.

No obstante, los avances en la legislación laboral costarricense asociados también a las luchas sindicales, las condiciones laborales que crea la producción bananera están aún lejos de ser las más adecuadas para los trabajadores. Recientemente, un diagnóstico elaborado para la Asociación de Servicios de Promoción Laboral (ASEPROLA) sobre la situación laboral en las zonas bananeras del Caribe de nuestro país señala que

... si bien se han superado las condiciones de trabajo infrahumanas que afrontaron los trabajadores bananeros de principios del siglo pasado tales como el paludismo, la fiebre amarilla, la muerte por carencia de sueros antiofídicos y el maltrato físico, persiste la lucha por lograr mejores salarios, condiciones sanitarias, educativas, recreativas y de vivienda, con el agravante de presenciar a partir de las dos últimas décadas del siglo $\mathrm{XX}$ un retroceso en las conquistas de los trabajadores. (Banuett, 2003:4).

Lo anterior está relacionado en parte con el hecho de que, tras muchos años de investigación metódica, en la década de los setenta las compañías bananeras habían logrado optimizar la eficiencia del trabajo en diversas operaciones $y$ esto se vio facilitado por la continuidad y el carácter cíclico del cultivo. Así, las tasas de remuneración del trabajo a destajo se fueron estableciendo en relación con las metas de la productividad. Por eso, aun cuando las luchas sindicales en Costa Rica y Panamá lograron que se establecieran salarios mínimos por jornadas 
de ocho horas, estos fueron definidos a partir de los costos y las metas de productividad establecidas por las corporaciones bananeras (Ellis, 1983: 206-232).

Además, a fines de los años ochenta las compañías transnacionales y el gobierno costarricense promueven el llamado Plan de Fomento Bananero a partir del cual se busca reactivar la expansión bananera. Y para esto se facilita a las empresas bananeras: una exención de impuestos y regalías fiscales, la posibilidad de ampliar las áreas de cultivo y en la práctica se promueve la desregulación de la normativa ambiental y laboral (Arias y otros, 1999: 58-60).

Ese nuevo contexto de presión empresarial por bajar los costos de producción ${ }^{11}$ aunado al debilitamiento de los sindicatos explica situaciones como las siguientes:

$\diamond$ que las jornadas de trabajo en las bananeras puedan alcanzar las 12 ó 13 horas diarias cuando hay corta de banano,

$\diamond$ que se haya vuelto a las prácticas de salario a destajo, se paga por hectárea o por hora dependiendo de la tarea ${ }^{12} y$

11 En relación con las normativas laborales se ha dado una tendencia hacia la desregulación y flexibilización laboral, es decir una adaptación de las condiciones laborales a las necesidades del mercado y la producción. Esta se manifiesta en la variedad de formas de contratación que se le permite a las empresas entre las que se destacan: el contrato por selección, (solo por tres meses) la sub-contratación, el contrato de arrimado y el contrato por factura. Estas prácticas implican cada vez una menor responsabilidad de la empresa por la estabilidad laboral, la seguridad y el salario del trabajador (Banuett, 2003:2-7).

12 Considerando el empleo generado por las compañías bananeras transnacionales y nacionales en conjunto, las tareas y categorías ocupacionales de las plantaciones bananeras están organizadas como divisiones $y$ dentro de ellas se encuentran las siguientes áreas ocupacionales: fincas, empacadoras, transporte, muelle, ingeniería y mantenimiento, servicios y administración. El $72 \%$ de los empleos que se generan se ubican en las fincas (48\%) y las empacadoras (23\%). Dentro del trabajo de las fincas, en la mayor parte de las plantaciones se distinguen cinco categorías de tareas que se realizan antes de la cosecha y se denominan como limpieza, drenaje, aplicación de fertilizantes, control de $\diamond$ los beneficios sociales dependan del tipo de contrato que se establece ${ }^{13}$.

Aunque se afirma que los salarios pagados en la actividad bananera son superiores a los del resto del sector agrícola no compensan el desgaste físico y las malas condiciones a las que se ven expuestos algunos de sus trabajadores (Arias y otros, 1999: 61) ${ }^{14}$. Por otro lado, las nuevas formas de contratación favorecen la inestabilidad laboral y la discriminación de los inmigrantes ${ }^{15}$. Se calcula que solo un $30 \%$ de

enfermedades y protección. Una sexta categoría es la cosecha misma y la séptima es el transporte. En la empacadora las tareas incluyen: el desgaje de los racimos, el lavado, la selección, la colocación de la etiqueta, el empaque y el engrape (Ellis, 1983: 215, 223-227).

13 En la actividad bananera, mientras en 1975 el salario por hora era de 0,42 centavos de dólar a julio del año 2007 es de $\$ 1,67$ y por un puesto similar hoy en la actividad turística se paga $\$ 1,91$ por hora.

14 De hecho aún en la década del 1990 se ha dicho que el promedio salarial en la actividad es de $\$ 250$, que es relativamente más alto que el de otras actividades agrícolas (Arias y otros, 1999: 61). Pero también, el alto costo social $y$ ambiental de la actividad ha sido objeto de múltiples denuncias de las Ong y sindicatos que trabajan con el sector. Se han aportado estudios que muestran que este tipo de cultivo tiene impactos en la salud humana de quienes realizan algunas de sus tareas y también provoca contaminación de los suelos y el aire. Entre los efectos señalados en los trabajadores de la empacadora, están las lesiones en la piel y los ojos causadas por el contacto continuo de la humedad y cerca de sustancias químicas tales como el sulfato de aluminio y el funguicida tiabenzanol. Otro de los indicadores utilizados es el hecho de que la actividad bananera utiliza el 35\% de toda la cantidad de plaguicidas que Costa Rica importa anualmente (Vargas, 1998: 6).

15 Bourgois, evidenció la forma en que en los años veinte se produjo en las plantaciones una división del trabajo en torno a la etnicidad según la cual las tareas de limpieza en aquél tiempo más duras era trabajo de latinos mientras las tareas más técnicas, que requerían experiencia y menos duras fueron "trabajo de negro". Y casi sesenta años después, en los años 1982-1984, Bourgois señala que persiste en las bananeras "una jerarquía laboral de apartheid de facto" (Bourgois, 1994: 17, 121-123). 
los trabajadores bananeros de la zona atlántica tiene estabilidad laboral y el resto deambula por la zona (Banuett, 2003).

\subsection{LA PRECARIEDAD LABORAL DE LOS SERVICIOS TURÍSTICOS}

Si se ven los datos del último censo sobre el empleo en la actividad de turismo ${ }^{16}$ se tiene que, para la región huétar atlántica esta genera el $8 \%$ de puestos mientras que en el distrito de Cahuita alcanza casi el 20\% ${ }^{17}$. Dado que a partir del censo nacional algunas actividades vinculadas al turismo pueden no ser registradas como tales se buscó un acercamiento más exhaustivo y específico con las comunidades de interés y se aplicó, en el año 2003 una encuesta de hogares de Puerto Viejo (Molina, 2003). Con este instrumento se determinó que casi el 30\% de personas ocupadas tenían un empleo ligado al turismo. Este resultado coincidió con la encuesta de hogares llevada a cabo por FLACSO en Cahuita según la cual el $27 \%$ de las personas ocupadas tienen empleos en la actividad turística (Van Der Duim y otros, 2003:114). Estando la actividad turística en expansión en la zona conviene preguntarse ¿cuáles son las características del empleo generado y si el mismo constituye un empleo de calidad?

El desgaste físico y el ambiente laboral del sector de servicios turísticos es cualitativamente

El décimo informe del Estado de la Nación muestra cambios importantes en la estructura del empleo a nivel nacional, evidenciando un incremento de los ocupados en actividades del sector terciario en contraste con una pérdida de la participación relativa del sector agrícola en la generación del empleo. Mientras la rama de servicios pasó de representar un $48 \%$ en 1990 a un $62 \%$ en el año 2003, el empleo en el sector agrícola pasó de un 19 a 14\% en esos mismos años. El turismo con una tasa de crecimiento anual de $8 \%$ entre 1984-2000, (superior a la media nacional de $3,5 \%$ ) es uno de los sectores más dinámicas de la economía.

17 A pesar de lo anterior, en esa región, el sector agrícola sigue teniendo mayor peso que otros sectores (34\%) como generador de empleo pero la tendencia nacional al incremento de los ocupados en las actividades de servicios es también una realidad (Estado de la Nación, 2004:164). diferente de los requerimientos del trabajo en las plantaciones bananeras, al menos en algunas labores del campo y del empaque de la fruta. Esto porque el método de trabajo en las bananeras está organizado de forma similar al trabajo en serie desarrollado en la manufactura.

Las corporaciones bananeras han desarrollado investigaciones metódicas para optimizar los rendimientos en las diversas operaciones involucradas con el ciclo productivo y el pago de la fuerza de trabajo contratada responde a cálculos de costo/beneficios muy detallados $y$ orientados a determinadas metas de productividad $^{18}$.

Para aportar evidencia empírica a favor de la segunda tesis propuesta, se define el empleo precario como aquel que no otorga las condiciones establecidas como mínimas en la legislación costarricense.

Se propone para esto un abordaje desde los negocios turísticos por lo que se realizó una encuesta a 89 asalariados de 101 negocios turísticos de Cahuita y Puerto Viejo.

Una de las características más notorias de los resultados encontrados es la importancia de la participación femenina y juvenil en el empleo turístico, en contraste con otras actividades o segmentos laborales. En relación con lo primero, se encontró que el $52 \%$ de los empleados son

18 El mercado de los servicios turísticos, no escapa de la lógica de acumulación capitalista pero su expansión en nuestro país es mucho más reciente. Hasta se podría hacer el paralelo de la etapa actual de expansión turística con los primeros veinte años de la expansión bananera, donde el capital extranjero es el que "abre el camino" en el nuevo sector dinámico. Las lógicas de acumulación en el sector empresarial turístico en nuestro país han sido muy poco estudiadas pero es claro que hay desarrollos turísticos de grandes complejos hoteleros y aglomeraciones de pequeños negocios turísticos. En el caso de Cahuita y Puerto Viejo, se trata de pequeñas empresas turísticas que en su mayoría ofrecen uno o dos servicios turísticos tales como hospedaje, venta de comida, souvenir, información turística entre las principales. Tienen un promedio de dos empleados y una ganancia promedio de 275 mil colones mensuales. La principal forma en que han atraído sus clientes es por medio del contacto directo o la recomendación que hacen unos clientes a otros (Molina y otros, Encuesta de establecimientos turísticos Cahuita-Puerto Viejo, 2001). 
mujeres, lo cual contrasta con otros sectores, como el tradicional y el estatal, que suelen ser menos accesibles para las mujeres. Situación particularmente cierta para el caso de la producción bananera en la que si bien las empresas prefieren contratar mujeres en el empaque la proporción de estas sigue siendo menor en relación con el empleo masculino.

Por otro lado, el promedio de edad de los empleados en turismo para ambas comunidades es de 33 años. Otras de las características del sector empleado son las siguientes: el 61\% son extranjeros, en su mayoría (66\%) trabajan como asalariados aunque un $22 \%$ son familiares no remunerados. El promedio de trabajar en el negocio es de menos de tres años.

Para analizar la precariedad del empleo se utilizaron tres indicadores: el salario, el acceso al seguro social y la estabilidad laboral. De manera que puede definirse como empleo precario aquel que no cumple con el salario mínimo por hora según cada ocupación, que otorga el seguro social $y$, finalmente, que no garantiza algún grado de estabilidad laboral, ya sea por un contrato escrito o por un acuerdo verbal entre las partes (Herradora, Molina y Núñez, 2002:20).

A partir de un análisis elemental de estadística descriptiva se obtuvo que las dos condiciones que afectan a más empleados del turismo en los negocios de Cahuita y Puerto Viejo son: no contar con el pago del seguro social $(71 \%$ de encuestados) ${ }^{19}$ y recibir un salario por hora inferior al establecido por ley (42\%). En contraste con la ausencia de estabilidad laboral que afecta solo al $22 \%$ de trabajadores.

Uno de los hallazgos más interesantes del estudio fue evidenciar que la precariedad del empleo turístico se comporta diferencialmente según sexo y edad. En el cuadro se resumen los datos según sexo. Se puede notar que solo en el caso del acceso al seguro social se obtuvo una relación estadísticamente significativa según sexo. Esto significa que ser hombre o mujer tiene relevancia respecto del acceso al seguro social en el empleo de Cahuita y Puerto Viejo. Y en este caso ser mujer lo favorece.

CUADRO

EMPLEOS PRECARIOS SEGÚN SEXO EN ASALARIADOS DE CAHUITA Y PUERTO VIEJO

\begin{tabular}{|c|c|c|c|c|c|c|c|c|c|c|c|c|}
\hline $\begin{array}{l}\text { ASALARIADOS } \\
\text { DEL TURISMO } \\
\\
\text { Sexo }\end{array}$ & \multicolumn{4}{|c|}{ SEGURO } & \multicolumn{4}{|c|}{ SALARIO POR HORA } & \multicolumn{4}{|c|}{ TIPO DE CONTRATACIÓN } \\
\hline \multirow{2}{*}{ Sexo } & \multicolumn{2}{|c|}{$\begin{array}{c}\text { CONDICIÓN } \\
\text { PRECARIA } \\
\end{array}$} & \multirow[t]{2}{*}{ TOTAL } & \multirow[t]{2}{*}{$\begin{array}{c}\text { SIGNIFI- } \\
\text { CANCIA }\end{array}$} & \multicolumn{2}{|c|}{$\begin{array}{l}\text { CONDICIÓN } \\
\text { PRECARIA }\end{array}$} & \multirow[t]{2}{*}{ TOTAL } & \multirow[t]{2}{*}{$\begin{array}{r}\text { SIGNIFI- } \\
\text { CANCIA }\end{array}$} & \multicolumn{2}{|c|}{$\begin{array}{c}\text { CONDICIÓN } \\
\text { PRECARIA } \\
\end{array}$} & \multirow[t]{2}{*}{ TOTAL } & \multirow[t]{2}{*}{$\begin{array}{l}\text { SIGNIFI } \\
\text { CANCIA }\end{array}$} \\
\hline & No & Sí & & & No & Sí & & & No & Sí & & \\
\hline Hombres & $24 \%$ & $48 \%$ & $41 \%$ & 0.37 & 40 & 39 & $39 \%$ & 945 & 37 & 52 & $41 \%$ & 222 \\
\hline Mujeres & $76 \%$ & $52 \%$ & $59 \%$ & 0.37 & $60 \%$ & $61 \%$ & $61 \%$ & 945 & 62 & 48 & $61 \%$ & 222 \\
\hline
\end{tabular}

Fuente: Elaboración propia con datos de la encuesta de asalariados del turismo diseñada y aplicada por la autora junto con Michael Herradora y Edgar Núñez, 2002.

19 Se complementó con 151 encuestas solo en hogares de Puerto Viejo. En relación con el acceso a los beneficios sociales, los datos se ampliaron con información de todos los miembros de cada hogar, lo que permitió visualizar el acceso a estos, independientemente de que la ocupación sea en el sector turístico o no. La encuesta aplicada mostró que el 57\% de ocupados no gozaba de ningún beneficio social (seguro, aguinaldo o vacaciones) con lo que se planteó la cuestión de la desprotección laboral como posible situación generalizada en las diversas ocupaciones (Molina, Encuesta hogares de Puerto Viejo, 2003). 
Así, mientras que el $76 \%$ de las mujeres empleadas en turismo gozan de seguro social, en el caso de los varones, el porcentaje alcanzó solo al 24\%. En el caso del salario por hora y la contratación, los porcentajes de precariedad y no precariedad laboral no son relevantes estadísticamente como lo muestran los niveles de significancia. Cuando el análisis se aplica tomando en cuenta la edad de los asalariados se obtiene que tanto la precariedad laboral por trabajo inestable como el pago de salarios por hora inferiores constituye una tendencia más acentuada en los empleados más jóvenes. Lo anterior llama la atención sobre diferentes tipos de precariedad laboral de acuerdo con el género $y$ la edad.

Es importante destacar dos aspectos en los cuales el empleo generado por el turismo en Talamanca no difiere mucho del empleo generado por las transnacionales bananeras: en el tipo de ocupación predominante $y$ en las condiciones de inestabilidad laboral. En las dos comunidades estudiadas se encontró que la mayoría de puestos generados se ubican en tres ocupaciones que son salonero, jardinero, mucama o encargada de limpieza y cocinero. En las transnacionales las principales ocupaciones son peón agrícola, bodeguero y empaque. Esto significa que en ambos casos el empleo generado es de baja calificación y los salarios por hora no difieren sustancialmente en ambas ramas o sectores económicos.

Por otro lado, existen lógicas de expansión globales que afectan tanto el empleo del sector turismo como el de las corporaciones bananeras y son las tendencias a la flexibilización y desregulación laboral. En Cahuita y Puerto Viejo por ejemplo, se encontró que el $52 \%$ de empleados en turismo tenían un año o menos de haber iniciado en el trabajo actual $y$ en el caso de los propietarios de negocios el $55 \%$ de estos tenían cinco años o menos de haber puesto ese negocio. Además analizando la historia laboral de algunos empleados se encontró que, aunque tenían varios años de trabajar en el sector turístico habían pasado al menos por tres o cuatro locales en los últimos cinco años. Ya se mencionó que la inestabilidad laboral afecta directamente las condiciones salariales y el acceso a los beneficios sociales.

\section{CONCLUSIONES}

La investigación realizada permite hacer algunas reflexiones, a manera de conclusiones, con respecto a tres aspectos: en primer lugar, sobre los cambios ocurridos en la historia reciente de las dos comunidades de estudio Cahuita y Puerto Viejo; en segundo lugar, sobre las principales lógicas que incidieron sobre esos cambios y por último, algunos comentarios sobre el estudio de la relación entre lo local y lo global.

\subsection{SOBRE LAS TRANSFORMACIONES DE CAHUITA Y PUERTO VIEJO}

Se argumentó que en los poblados estudiados se produjo una transformación socioterritorial que dio como resultado el paso de una sociedad local agrícola a una sociedad basada en la venta de los servicios turísticos. Y se destacaron tres efectos socioespaciales a partir de la expansión de la actividad turística: a) el ensanchamiento territorial de las comunidades que, han pasado a visualizarse y funcionar como micro-región turística en vez de las comunidades o colonias agrícolas, b) la llegada de los nuevos empresarios nacionales o extranjeros que han generado una aglomeración de pequeñas y medianas empresas con un potencial de cluster turístico; $y$ c) el desplazamiento de gran parte de la población de origen antillano que había poblado el distrito.

Se argumentó, además, que estas transformaciones han conllevado impactos desiguales sobre el territorio, la población y la estructura social asentada en la zona. De manera que, el acceso a los servicios turísticos ha segmentado el territorio y la población. Uno de los indicadores de lo anterior es la importancia del empleo que ha generado la expansión del turismo como actividad principal en la faja costera. Este emplea aproximadamente un $30 \%$ de la fuerza de trabajo ocupada en Cahuita y Puerto Viejo.

El proceso de tercerización de la economía entendido como un fortalecimiento del sector servicios, ha hecho pensar a algunos que el aumento de las ocupaciones en este campo 
son sinónimo de desarrollo local. No obstante, un repaso sobre la situación laboral de los empleados bananeros y los empleados en turismo mostró algunos puntos de comparación y la necesidad de afinar el análisis en estudios posteriores.

Si bien, es un hecho que el trabajo en servicios turísticos es menos agotador y desgastante físicamente comparado con la producción bananera algunas de las ocupaciones más requeridas son comunes en ambas actividades como por ejemplo, la de peón agrícola equivalente a jardinero, la de limpieza o la de cocinero. En términos salariales y de acceso a beneficios sociales en ambas industrias la tendencia más clara es a un importante grupo que no recibe ni el salario mínimo y una gran proporción que sufre de la inestabilidad laboral y la desregulación. Si comparando el empleo agrícola con el turístico suele verse el segundo como un empleo de mayor calidad, cuando se analiza el cumplimiento de este último con la legislación laboral se nota que es un empleo precario. Esto, en la medida en que, como evidenció la investigación, hubo sectores importantes de los empleados que reciben menos que el salario por hora mínimo, que no acceden al seguro social y no gozan de estabilidad laboral.

\subsection{SOBRE LAS PRINCIPALES LÓGICAS SOCIALES QUE INCIDIERON EN LAS TRANSFORMACIONES}

La investigación realizada permitió acercarse a las dos comunidades costeras identificando el conjunto de lógicas que operan dentro de un territorio $y$ evidenciando tres elementos que han jugado un papel determinante como factores condicionantes y condicionados a la vez. Estos elementos son el territorio, la organización productiva, $y$ la generación de empleo, todos ellos, por sus características podrían visualizarse como parte de los recursos internos de las comunidades en contraste con tres lógicas internacionales que impactaron fuertemente la historia de Cahuita: la expansión de la producción bananera, la reciente expansión de los servicios turísticos y los flujos migratorios ligados a ambas.

\subsection{SOBRE LA RELACIÓN ENTRE LO GLOBAL Y LO LOCAL}

Producto de las transformaciones del capitalismo, manifiestas desde fines de los años setenta pero sobre todo a partir de las dos últimas décadas del siglo pasado, han surgido muchos esfuerzos por interpretar el cambio social ocurrido. Desde distintas perspectivas se ha tomado el concepto de globalización para designar una nueva fase en el desarrollo capitalista y se ha planteado una nueva relación entre procesos locales y globales. Estos esfuerzos si bien tienen el mérito de re-situar los problemas en un nuevo contexto mundial, lo cierto es que en algunos casos generan conceptos cargados de generalidad y ambigüedad. Ese es el caso de la tensión existente entre lo local y lo global, la cual fue abordada en los años 1940-1950 como el estudio de las relaciones entre factores externos e internos al estado nación. Actualmente se utiliza la noción de lo global aludiendo no solo al sistema mundial sino también a las lógicas económicas como las fuerzas determinantes del cambio social. Teniendo en cuenta lo anterior, en esta investigación se utilizó el concepto de lógicas históricas y sistémicas para aludir a las lógicas internas en contraposición a las lógicas externas. Y se llegó a dos conclusiones importantes. Por un lado, que no hay lógicas que sean sistémicas o parciales per se, pues, dependiendo de la confluencia de diversos procesos sociales unas variables se vuelven más determinantes que otras, en distintas fases, para explicar el rumbo que toma una sociedad local. Un ejemplo, de esto lo constituye la organización productiva de las comunidades que como se ha señalado responde tanto a las condiciones geográficas y los recursos de la zona, como a las lógicas implantadas desde fuera y ligadas al desarrollo de la industria bananera mundial o posteriormente del turismo.

Y por otro lado, es claro que ambas lógicas (históricas y sistémicas) no pueden ser disociadas porque lo comunitario se construye precisamente del encuentro entre sus dinámicas. Por lo tanto, hablar de lo glocal permite enfatizar en que los procesos concretos que se visualizan en un territorio están emparentados con procesos de mayor alcance y también es una 
forma de decir que lo local, hoy más que nunca, es la forma específica en que los procesos societales se expresan en determinados espacios.

\section{BIBLIOGRAFÍA CONSULTADA}

Arias Alfaro, Mirzeth; Ferreto Alvarado, Olga Marta; Muñoz Loaiza, Gioconda. "Autopercepción de la salud ocupacional de las obreras y los obreros de Matina, Limón”. [Tesis de Grado Escuela de Antropología y Sociología]. Facultad de Ciencias Sociales, Universidad de Costa Rica, 1999.

Acuña, Marvin y Ruiz, Keinor. "Contribución del desarrollo turístico sobre el empleo rural no agrícola en Costa Rica". En: Revista Economía y Sociedad 14, septiembre-diciembre, 2000: 85-107.

Arocena, José. El desarrollo local: un desafío contemporáneo. Caracas: Centro Latinoamericano de Economía HumanaUniversidad Católica del UruguayEditorial Nueva Sociedad, 1995: 19-55.

Banuett, Marcelle. Situación laboral en las zonas bananeras del Caribe costarricense. Consultoría para Aseprola y el Foro de Emaús. San José, Costa Rica, 2003

Beck, Ulrico. ¿Qué es la globalización? Falacias del globalismo, respuestas a la globalización. Barcelona: PAIDÓs, 1997: 77-83.

Bourgois, Philippe. Banano, etnia y lucha social en Centro América. Primera Edición en español. San José: Editorial Departamento Ecuménico de Investigaciones (DEI), 1994.

Carcanholo, Reinaldo. "Sobre la evolución de las actividades bananeras en Costa Rica. Estudios Sociales Centroamericanos 19, enero-abril.
Confederación Centroamericana de Ciencias Sociales (CSUCA), 1978:143-206.

Casey Gaspar, Jeffrey. "Limón: 1880-1840. Un estudio de la industria bananera en Costa Rica”. Estudios Sociales Centroamericanos 23, mayo-agosto. CSUCA, 1979: 245-282.

Ellis, Frank. Las transnacionales del banano en Centroamérica. Primera Edición. Editorial EDUCA, 1983: 211-257.

Fonseca, Marvin y Weitzner, Viviane. "Comanejo y participación civil en las áreas protegidas: la experiencia del Parque Nacional Cahuita”. Gestión Ambiental Descentralizada. Convenio Bilateral de Desarrollo Sostenible Costa Rica- Holanda: INCO, Costa Rica, 1999:199-249.

Fürst, Edgar y Hein, Wolfgang. Turismo de larga distancia y desarrollo regional en Costa Rica. Estudios sobre las relaciones económico-ecológicas entre turismo y desarrollo sostenible en los ámbitos globales, nacionales y microrregionales. Heredia: Centro Internacional de Política Económica para el Desarrollo Sostenible (CIMPE) de la Universidad Nacional Autónoma, con apoyo de la cooperación alemana, 2001: 25-143.

Goluboay, Juan Miguel y Vega, Herbert: "La actividad bananera en Costa Rica". FLACSO y CEDAL. Cambio y continuidad en la economía bananera. Editorial FLACSO, 1987: 131-163.

Herradora, M.; Núñez E; Molina, Eugenia. "Turismo y empleo en Cahuita $y$ PuertoViejo: Una aproximación a formas de precarización laboral". San José: Informe final de investigación para el Diplomado en Investigación del Desarrollo Económico Local de la Facultad Latinoamericana de Ciencias Sociales, FLACSO, 2002. 
Hiernaux-Nicolás, Daniel; Cordero, Allen; Van Duynen, Luisa. "Imaginarios sociales y turismo sostenible". San José: Cuaderno de Ciencias Sociales 123 de la FLACSOSede Costa Rica, 2002.

Lathrop, Guillermo y Perez Sáinz, Juan Pablo. Desarrollo económico local en Centroamérica. Estudios de comunidades globalizadas. San José: FLACSO, 2004: 221-256.

Molina, Eugenia. "Un estado de la cuestión sobre turismo, identidad y desarrollo local". Informe de investigación del proyecto: "El potencial de los espacios locales para la resolución de problemas del desarrollo y la conformación de identidades". San José: Instituto de Investigaciones Sociales de la Universidad de Costa Rica, 2005.

Palmer, Paula. "Wa pin man" La historia de la costa talamanqueña de Costa Rica, según sus protagonistas. San José: Editorial de la Universidad de Costa Rica, 2000.

Pérez Sáinz, Juan Pablo; Rivera Araya, Roy et ál. Encuentros inciertos. San José de Costa Rica: FLACSO, 2000: 7-52.

Piedrahita, Jaime. "El arrecife coralino de Punta Cahuita. Origen de un pueblo y un
Parque Nacional”. Cooperación Técnica Bilateral Holanda-Costa Rica, Proyecto Namasol, Costa Rica, 1997: 8-20.

Sojo, Carlos y Pérez Sáinz, Juan Pablo. "Reinventar lo social en América Latina". Carlos Sojo (Editor), América Latina: Temas y desafíos para las politicas públicas. San José de Costa Rica: FLACSO, 2002: 16-64.

Van Der Duim, René; Caalders, Janine; Cordero, Allen y otros. El desarrollo del turismo sostenible. Los casos de Manuel Antonio y Texel. San José de Costa Rica: FLACSO, 2003: 95-118.

Viales, Ronny. "La colonización agrícola de la Región Atlántica (Caribe) costarricense entre 1870 y 1930. El peso de la política agraria liberal $y$ de las diversas formas de apropiación territorial". Anuario de Estudios_Centroamericanos 27 (2). San José, 2001: 57-100.

. Después del enclave (1927-1950). Primera edición. San José: editorial Universidad de Costa Rica, 1998.

Wallerstein, Emmanuel. El moderno sistema mundial. México: Siglo XXI, 1979: 399502. 